\title{
Adsorption Sites of Hydrogen Atom on Pure and Mg-Doped Multi-Walled Carbon Nanotubes
}

\author{
A. A. Al-Ghamdi, ${ }^{1}$ E. Shalaan, ${ }^{1}$ F. S. Al-Hazmi, ${ }^{1}$ Adel S. Faidah, ${ }^{1}$ \\ S. Al-Heniti, ${ }^{1}$ and M. Husain ${ }^{2}$ \\ ${ }^{1}$ Physics Department, Faculty of Science, King Abdulaziz University, Jeddah 21589, Saudi Arabia \\ ${ }^{2}$ Physics Department, Jamia Millia Islamia University, New Delhi 110025, India
}

Correspondence should be addressed to A. A. Al-Ghamdi, aghamdi90@hotmail.com

Received 19 May 2012; Accepted 22 July 2012

Academic Editor: Xuedong Bai

Copyright ( 2012 A. A. Al-Ghamdi et al. This is an open access article distributed under the Creative Commons Attribution License, which permits unrestricted use, distribution, and reproduction in any medium, provided the original work is properly cited.

\begin{abstract}
Hydrogen adsorption sites on pure multiwalled carbon nanotube (MWCNT) and Mg-doped MWCNTs material system have been investigated using molecular dynamics (MD) simulations as well as quantum chemical calculations. Through combining MWCNTs with $\mathrm{Mg}$, the hydrogen adsorption sites energy on this Mg-MWCNTs system is found to be larger than that of the pure MWCNTs. Additionally, it was found that, through Mg-doping, new adsorption sites for hydrogen molecules are created in comparison with undoped nanotubes. It is also found that $\mathrm{H}$ atom is preferably adsorbed at every place near magnesium atom.
\end{abstract}

\section{Introduction}

Hydrogen is an attractive alternative energy carrier for future fuel needs. The use of hydrogen as a fuel requires development in different industry segments, including production, delivery, and storage. One of the most critical factors facing hydrogen economy is transportation and on-vehicle storage of hydrogen [1-9]. The major contribution to the problem is from low gas density of hydrogen.

Metal hydrides are specific combinations of metallic alloys, which possess the unique ability to absorb hydrogen (hydrogenation) and release it later (dehydrogenation), either at room temperature or on heating. The percentage of gas reversibly absorbed to weight of the metal is around 2\%, but hydrides offer a valuable solution to hydrogen storage $[10,11]$. Magnesium hydride $\left(\mathrm{MgH}_{2}\right)$ is inexpensive and has a maximum storage capacity of $7.6 \mathrm{wt} \% \mathrm{H}_{2}$.

Various forms of carbon such as fullerene, nanotubes, graphene, and activated carbon with high surface area may be used for the storage of hydrogen. Single-walled carbon nanotubes can store $2.5-3 \mathrm{wt} \%$ hydrogen $[3,12]$. Research has focused on the areas of improving manufacturing techniques and reducing costs as carbon nanotubes move toward commercialization [3]. Others have proposed storing hydrogen in fullerenes or in activated carbon at low temperatures [12-15].

There are extensive scientific researches on the hydrogen storage via carbon nanotubes [16-30]. Unfortunately, widely varying reported weight percentages were emerged form those studies, leading to a scientific confusion that arises for two reasons: the experimental samples are always far from theoretically pure investigated samples, and the mechanisms of hydrogen adsorption are not yet understood [16-19].

Recently, McAfee and Poirier declare that hydrogen storage via carbon nanotubes requires exohedral adsorption in atomic form for which no more than one $\mathrm{H}$ atom adsorbate is bound per carbon nanotubes substrate $\mathrm{C}$ atom $[31,32]$. This yields a theoretical maximum storage capacity of $7.75 \%$, which is above the 2010 target, in which the US Department of Energy (DoE) has set a benchmark goal for on-board gravimetric hydrogen storage capacities of $6 \%$ by weight hydrogen by 2010 and $9 \%$ by 2015 [33].

However, combination of carbon nanotubes and metal hydrides in single nanostructure system may give a promising candidate for hydrogen storage.

Therefore, the aim of this research study is to investigate the hydrogen adsorption sites on Mg-doped MWCNT 


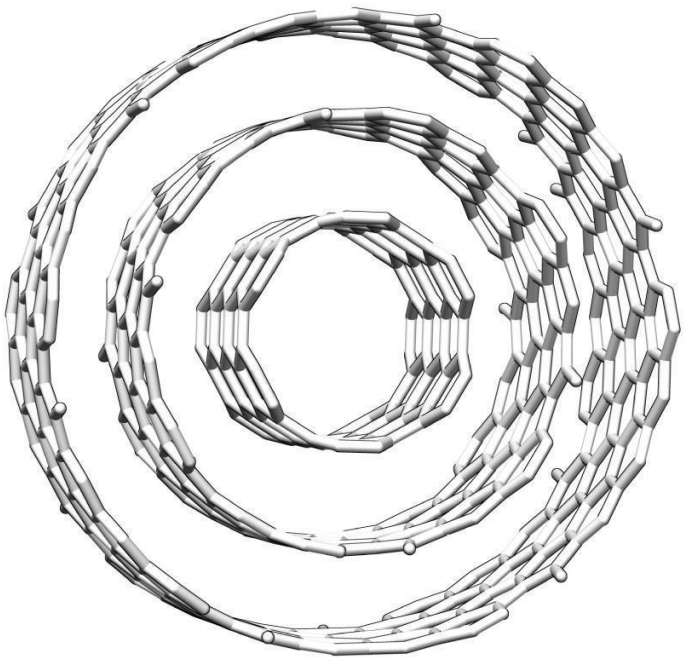

(a)

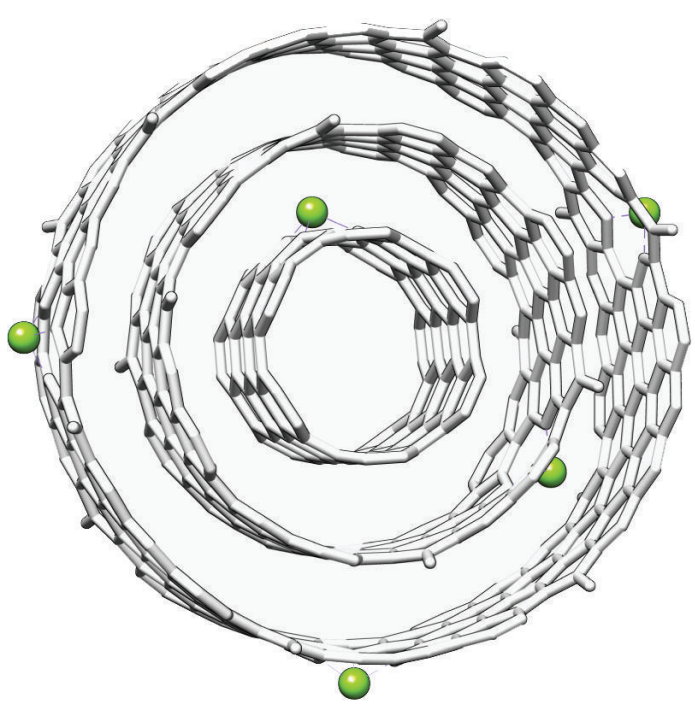

(b)

FIGURE 1: Top views of the fully relaxed structures of (a) a perfect arm-chair $(6,6)$ MWCNTs; (b) Mg-doped (6, 6) MWCNTs.

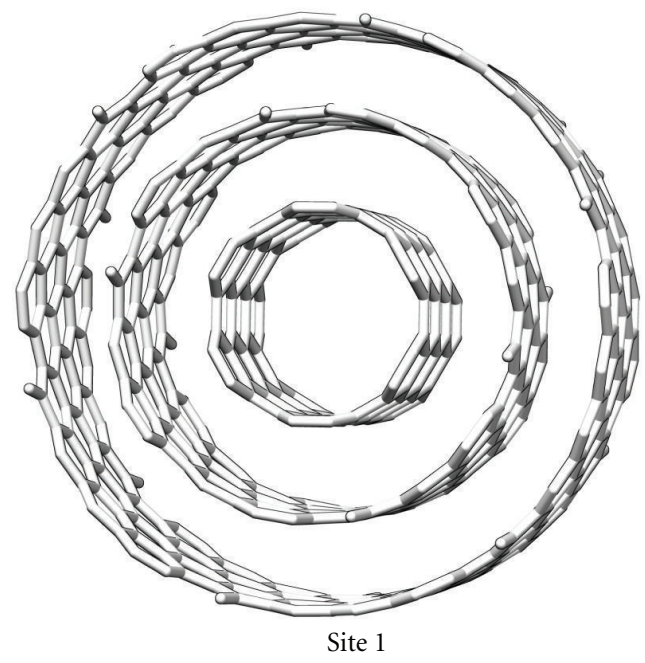

(a)

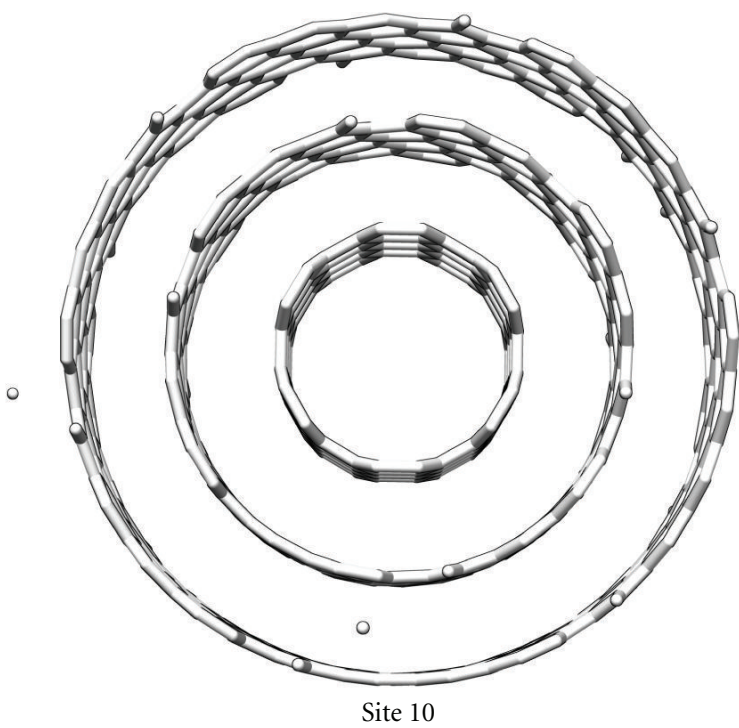

(b)

Figure 2: Top views of the fully relaxed structures of different adsorbed sites of $\mathrm{H}$ atom adsorbed to undoped arm-chair $(6,6) \mathrm{MWCNT}$.

(denoted Mg-MWCNTs) as a model material through the methodology of quantum mechanical (QM) calculations using general gradient approximation-density functional theory (GGA-DFT).

\section{Computational Methods}

In the current study, hydrogen atom has been simulated as adsorbate on pure MWCNTs and Mg-MWCNTs to find the energy adsorption sites and to investigate the preferential adsorption of the hydrogen atom onto MWCNTs and MgMWCNTs. The computational study was made using For cite and Adsorption locator in Accelrys Materials Studio software [34-36]. In order to investigate the adsorption of atomic hydrogen on MWCNTs, we perform a series of total energy calculations using adsorbate locator module ( $\max$ force $0.002 \mathrm{Ha} / \mathrm{A}$, energy $1 \times 10^{-5} \mathrm{Ha}$, max displacement $=$ $0.005 \mathrm{~A}$, and max step size $=0.3 \mathrm{~A}$ ) utilizing GGA:BLYP function. For substitutional doping, we replace, randomly, some carbon atoms by $\mathrm{Mg}$ atoms $(1.23 \% \mathrm{Mg} / \mathrm{C}$ by atom) followed by geometric optimization to study their effects on hydrogen adsorption using Forcite module with the following parameters: (energy $=2 \times 10^{-5} \mathrm{kcal} / \mathrm{mol}$, force $=0.001 \mathrm{kcal} / \mathrm{mol} / \mathrm{A}$, and displacement $=1 \times 10^{-5} \mathrm{~A}$ ). The geometry optimization process is carried out using an iterative process, in which the atomic coordinates are adjusted until the total energy of 


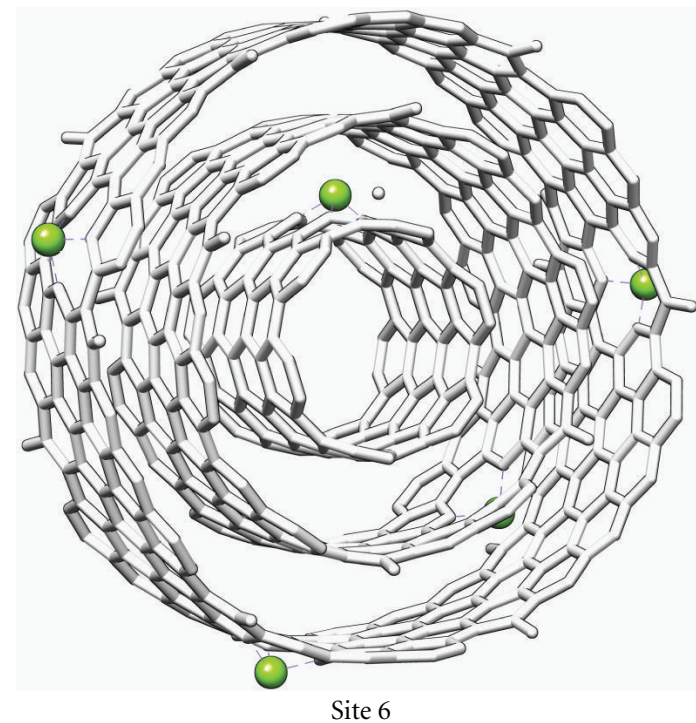

(a)

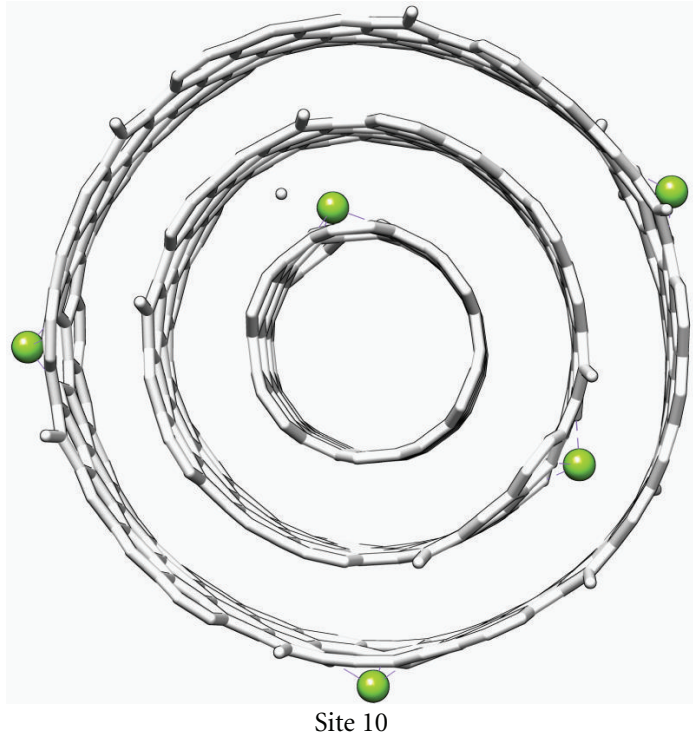

(b)

Figure 3: Top views of the fully relaxed structures of different adsorbed sites of $\mathrm{H}$ atom adsorbed to Mg-doped arm-chair $(6,6)$ MWCNT.

TABLE 1: Adsorption energy of $\mathrm{H}$ for many pure MWCNTs systems and Mg-MWCNTs systems.

\begin{tabular}{lcc}
\hline Structures & $\begin{array}{c}\text { MWCNTs systems } \\
\text { adsorption energy } \\
(\text { Kcal/mol) }\end{array}$ & $\begin{array}{c}\text { Mg-MWCNTs systems } \\
\text { adsorption energy } \\
(\text { Kcal/mol })\end{array}$ \\
\hline Site 1 & -2.01141053 & -2.03960997 \\
Site 2 & -0.84135214 & -2.03813645 \\
Site 3 & -0.8417632 & -0.90038241 \\
Site 4 & -0.81573871 & -0.86738694 \\
Site 5 & -0.79840829 & -0.86681624 \\
Site 6 & -0.78346106 & -0.86253083 \\
Site 7 & -0.72900349 & -0.87243483 \\
Site 8 & -0.84004276 & -0.84635485 \\
Site 9 & -0.72515658 & -0.84910777 \\
Site 10 & -0.51424081 & -0.84481357 \\
\hline
\end{tabular}

a structure is minimized. Geometry optimization is based on reducing the magnitude of calculated forces until they become less than preselected tolerance, $(0.01 \mathrm{eV} / \mathrm{A}$ in our case). The forces on the atoms are calculated from the potential energy expression and will, therefore, depend on the force field that is selected.

\section{Results and Discussions}

To obtain the configuration of the hydrogen adsorption on the MWCNT system, first, we fully optimized the geometry of the MWCNT before placing Mg atoms on it. Next, we simulated the doping of $\mathrm{Mg}$ on various positions on the MWCNT.

We studied two types of materials as depicted in Figure 1: perfect MWCNTs (Figure 1(a)) and Mg-MWCNT (Figure 1(b)) to study Mg effects on hydrogen adsorption sites. MWCNTs were built with the following parameters: $(\mathrm{CNT}(6 \times 6))$ with diameter $=8.14 \mathrm{~A}$, length $=9.84 \mathrm{~A}$, the number of walls is 3 , and the wall separation is $3.347 \mathrm{~A}$ ).

The fully optimized atomic structure of a perfect semiconducting arm-chair $(6,6)$ MWCNT and Mg-MWCNTs containing a single $\mathrm{H}$ atom adsorbate at different sites is shown in Figures 2 and 3, respectively.

We tried to find the effects of Mg-doping on the hydrogen adsorption behavior of Mg-MWCNTs. The calculations of the adsorption energies indicated that two distinct adsorption sites are created by the doped $\mathrm{Mg}$ atoms: one is the region where the distribution status of electrons is influenced by the doped $\mathrm{Mg}$ atoms (region 1) and the other one is the region of positively charged $\mathrm{Mg}$ atoms (region 2), created due to the transfer of electrons from $\mathrm{Mg}$ atoms to MWCNTs. When hydrogen is adsorbed on top of a $\mathrm{C}$ atom in carbon nanotube, the covalent $\mathrm{C}-\mathrm{H}$ bond is formed, and the neighboring $\mathrm{C}-\mathrm{C}$ binding is weakened. When hydrogen is adsorbed on top of $\mathrm{Mg}$ atom (region 2) in the $\mathrm{Mg}$-doped carbon nanotubes, $\mathrm{H}$ atom transfers some electronic charges to $\mathrm{Mg}$ atom and forms coordination-like bond between them. While Mg-doped carbon nanotube (region 1) is an electron-rich structure, it has no tendency to accept electrons from adsorbed atoms, and it will form mainly ionic $\mathrm{Mg}-\mathrm{H}$ bond. Table 1 illustrates the adsorption energy of hydrogen atom for pure MWCNTs systems and Mg-MWCNT systems. The $\mathrm{H}$ adsorption energies for Mg-MWCNTs are larger than that of the pure MWCNTs. This result shows that the $\mathrm{H}$ adsorption capability of the Mg-doped system is superior to the pure MWCNT system.

\section{Conclusions}

We have shown through computational simulations that Mg-MWCNTs can be good hydrogen storage medium. This study suggests that a systematic increase of binding energy of 
hydrogen can be achieved by moderate substitutional doping of MWCNTs with $\mathrm{Mg}$ atoms.

\section{Acknowledgment}

This research is supported by the Deanship of Scientific Research (DSR), King Abdulaziz University, Strategic Unit under Grant no. 8-NAN185-3, Saudi Arabia, which is greatly appreciated.

\section{References}

[1] C. Carpetis, "Estimation of storage costs for large hydrogen storage facilities," International Journal of Hydrogen Energy, vol. 7, no. 2, pp. 191-203, 1982.

[2] A. Chambers, C. Park, T. K. Baker, and N. M. Rodriguez, "Hydrogen storage in graphite Nanofi bers," The Journal of Physical Chemistry B, vol. 102, no. 22, pp. 4253-4256, 1998.

[3] A. C. Dillon, K. M. Jones, T. A. Bekkedahl, C. H. Kiang, D. S. Bethune, and M. J. Heben, "Storage of hydrogen in singlewalled carbon nanotubes," Nature, vol. 386, no. 6623, pp. 377379, 1997.

[4] C. A. Kukkone and M. Shelef, "Hydrogen as an alternative automotive fuel," in Alternate Fuels, R. M. Bata, Ed., National Research Council and National Academy of Engineering, National Academies Press, Washington, DC, USA, 1992.

[5] G. Thomas, "Hydrogen Storage: state of the art," in Proceedings of the Basic Energy Sciences Workshop on Basic Research Needs for the Hydrogen Economy, Rockville, Md, USA, May 2003.

[6] M. A. DeLuchi, "Hydrogen vehicles: an evaluation of fuel storage, performance, safety, environmental impacts, and cost," International Journal of Hydrogen Energy, vol. 14, no. 2, pp. 81-130, 1989.

[7] F. Michel, H. Fieseler, G. Meyer, and F. Theiben, "On-board equipment for liquid hydrogen vehicles," International Journal of Hydrogen Energy, vol. 23, no. 3, pp. 191-199, 1998.

[8] E. Newson, T. H. Haueter, P. Hottinger, F. von Roth, G. W. H. Scherer, and T. H. Schucan, "Seasonal storage of hydrogen in stationary systems with liquid organic hydrides," International Journal of Hydrogen Energy, vol. 23, no. 10, pp. 905-909, 1998.

[9] V. N. Verbetsky, S. P. Malyshenko, S. V. Mitrokhin, V. V. Solovei, and Y. F. Shmal'ko, "Metal hydrides: properties and practical applications. Review of the works in CIS-countries," International Journal of Hydrogen Energy, vol. 23, no. 12, pp. 1165-1177, 1998.

[10] Y. Kojima, Y. Kawai, S. I. Towata, T. Matsunaga, T. Shinozawa, and M. Kimbara, "Development of metal hydride with high dissociation pressure," Journal of Alloys and Compounds, vol. 419, no. 1-2, pp. 256-261, 2006.

[11] W. H. Scholz, "Processes for industrial production of hydrogen and associated environmental effects," Gas Separation and Purification, vol. 7, no. 3, pp. 131-139, 1993.

[12] L. Schlapbach and A. Züttel, "Hydrogen-storage materials for mobile applications," Nature, vol. 414, no. 6861, pp. 353-358, 2001.

[13] S. Hirano, K. S. Young, A. Kwabena, and J. A. Schwarz, "The high surface area activated carbon hydrogen storage system," in Proceedings of the 1st International Conference on New Energy Systems and Conversions, Frontiers Science Series, no. 7, pp. 67-72, Yokohama, Japan, June 1993.

[14] J. Chen and F. Wu, "Review of hydrogen storage in inorganic fullerene-like nanotubes," Applied Physics A, vol. 78, no. 7, pp. 989-994, 2004.
[15] R. J. Ströbel, J. Garche, P. T. Moseley, L. Jörissen, and G. Wolf, "Hydrogen storage by carbon materials," Journal of Power Sources, vol. 159, no. 2, pp. 781-801, 2006.

[16] C. Liu, Y. Y. Fan, M. Liu, H. T. Cong, H. M. Cheng, and M. S. Dresselhaus, "Hydrogen storage in single-walled carbon nanotubes at room temperature," Science, vol. 286, no. 5442, pp. 1127-1129, 1999.

[17] R. T. Yang, "Hydrogen storage by alkali-doped carbon nanotubes-revisited," Carbon, vol. 38, no. 4, pp. 623-626, 2000.

[18] M. Hirscher, M. Becher, M. Haluska et al., "Are carbon nanostructures an efficient hydrogen storage medium?" Journal of Alloys and Compounds, vol. 356-357, pp. 433-437, 2003.

[19] L. Zhou, Y. Zhou, and Y. Sun, "Enhanced storage of hydrogen at the temperature of liquid nitrogen," International Journal of Hydrogen Energy, vol. 29, no. 3, pp. 319-322, 2004.

[20] A. Nikitin, H. Ogasawara, D. Mann et al., "Hydrogenation of single-walled carbon nanotubes," Physical Review Letters, vol. 95, no. 22, Article ID 225507, 4 pages, 2005.

[21] A. R. Muniz, M. Meyyappan, and D. Maroudas, "On the hydrogen storage capacity of carbon nanotube bundles," Applied Physics Letters, vol. 95, no. 16, Article ID 163111, 3 pages, 2009.

[22] A. Nikitin, X. Li, Z. Zhang, H. Ogasawara, H. Dai, and A. Nilsson, "Hydrogen storage in carbon nanotubes through the formation of stable C-H bonds," Nano Letters, vol. 8, no. 1, pp. 162-167, 2008.

[23] S. S. Han and H. M. Lee, "Adsorption properties of hydrogen on $(10,0)$ single-walled carbon nanotube through density functional theory," Carbon, vol. 42, no. 11, pp. 2169-2177, 2004.

[24] V. Barone, J. Heyd, and G. E. Scuseria, "Interaction of atomic hydrogen with single-walled carbon nanotubes: a density functional theory study," The Journal of Chemical Physics, vol. 120, no. 15, pp. 7169-7173, 2004.

[25] I. Cabria, M. J. López, and J. A. Alonso, "Adsorption of hydrogen on normal and pentaheptite single wall carbon nanotubes," European Physical Journal D, vol. 34, no. 1-3, pp. 279-282, 2005.

[26] S. H. Yang, W. H. Shin, J. W. Lee, S. Y. Kim, S. I. Woo, and J. K. Kang, "Interaction of a transition metal atom with intrinsic defects in single-walled carbon nanotubes," Journal of Physical Chemistry B, vol. 110, no. 28, pp. 13941-13946, 2006.

[27] A. J. Lachawiec Jr., G. Qi, and R. T. Yang, "Hydrogen storage in nanostructured carbons by spillover: bridge-building enhancement," Langmuir, vol. 21, no. 24, pp. 11418-11424, 2005.

[28] R. Zacharia, K. Y. Kim, A. K. M. Fazle Kibria, and K. S. Nahm, "Enhancement of hydrogen storage capacity of carbon nanotubes via spill-over from vanadium and palladium nanoparticles," Chemical Physics Letters, vol. 412, no. 4-6, pp. 369-375, 2005.

[29] F. H. Yang, A. J. Lachawiec Jr., and R. T. Yang, "Adsorption of spillover hydrogen atoms on single-wall carbon nanotubes," Journal of Physical Chemistry B, vol. 110, no. 12, pp. 62366244, 2006.

[30] L. Chen, A. C. Cooper, G. P. Pez, and H. Cheng, "Mechanistic study on hydrogen spillover onto graphitic carbon materials," Journal of Physical Chemistry C, vol. 111, no. 51, pp. 1899519000, 2007.

[31] J. L. McAfee and B. Poirier, "Quantum dynamics of hydrogen interacting with single-walled carbon nanotubes," The Journal of Chemical Physics, vol. 130, no. 6, Article ID 064701, 16 pages, 2009. 
[32] J. L. McAfee and B. Poirier, "Quantum dynamics of hydrogen interacting with single-walled carbon nanotubes: multiple $\mathrm{H}$ atom adsorbates," The Journal of Chemical Physics, vol. 134, no. 7, Article ID 074308, 19 pages, 2011.

[33] U. S. Department of Energy, Hydrogen Energy, http://www .hydrogen.energy.gov/.

[34] O. Ermer, "Calculation of molecular properties using force fields. Applications in organic chemistry," Structure \& Bonding, vol. 27, pp. 161-211, 1976.

[35] J. Barriga, B. Coto, and B. Fernandez, "Molecular dynamics study of optimal packing structure of OTS self-assembled monolayers on $\mathrm{SiO}_{2}$ surfaces," Tribology International, vol. 40, no. 6, pp. 960-966, 2007.

[36] S. Kirkpatrick, C. D. Gelatt Jr., and M. P. Vecchi, "Optimization by simulated annealing," Science, vol. 220, no. 4598, pp. 671-680, 1983. 

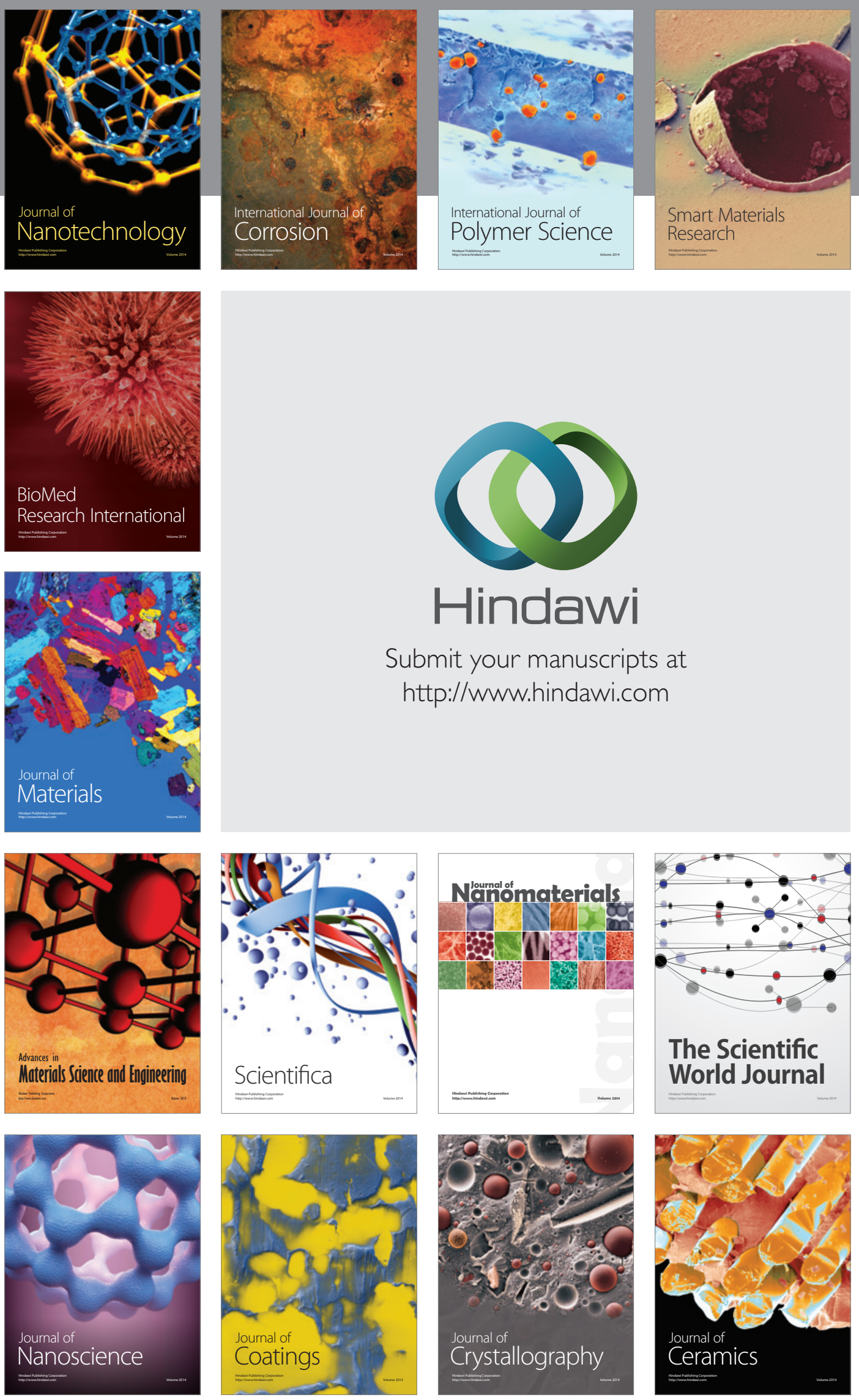

The Scientific World Journal

Submit your manuscripts at

http://www.hindawi.com

\section{World Journal}

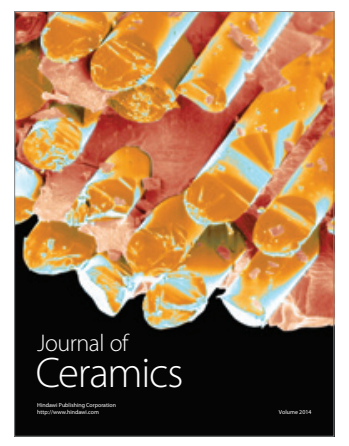

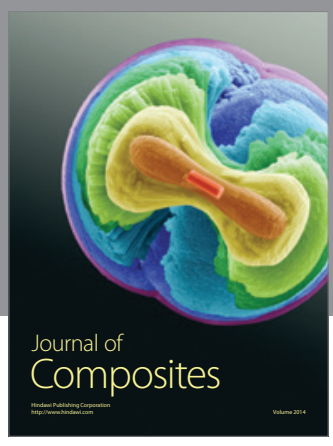
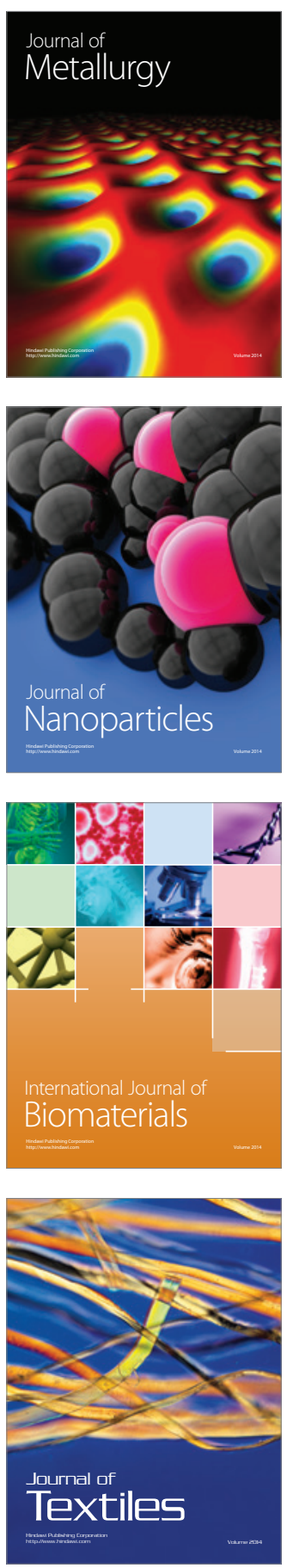